\title{
Novel Mass Spectrometry Mutation Screening for Contaminant Impact Analysis
}

Oak Ridge National Laboratory

Jul. 10, 2000

Annual Report

\section{Principal Investigator}

Dr. Winston Chung-Hsuan Chen

(423)-574-6895 (Phone)

(423)-576-2115 (Fax)

CHENC@ORNL.GOV

Oak Ridge National Laboratory

P. O. Box 2008

Oak Ridge, TN. 37831-6378

\section{Co-investigator}

Dr. Kai-Lin Lee

(423)-483-5771 (phone)

(423)-576-2115 (Fax)

Oak Ridge National Laboratory

P. O. Box 2008

Oak Ridge, TN. 37831-6378

\section{Other Contributors:}

Dr. Narayana Isola

Dr. Valeri V Golovlev

Dr. Richard Hurt

Dr. Chao-Yang Liu

\section{Graduate Students:}

John Kirk

Sma Lee 


\section{Research Objective:}

The objective of this program is to develop innovative mass spectrometry technology to achieve fast mutation analysis and to reveal the linkage between gene mutation and contaminants. Mass spectrometry has the potential to achieve very fast speed sample analysis. However, the poor mass resolution and low detection efficiency for long DNAs limit the broad application for mutation analysis. New innovative approaches for improving mass resolution and detection sensitivity needs to be pursued to help to achieve rapid DNA screening. Allele specific polymerase chain reaction (ASPCR) coupled with mass spectrometry can be very valuable for DNA mutation detection. This technology is expected to be applied to wildlife species such as fish for the genotoxic effect of hazardous waste to be assessed at DNA level.

\section{Research Progress and Implication:}

In this project, we have very significant achievement in mass spectrometry DNA analysis technology development which include the successful development of laser induce acoustic desorption and surface assisted desorption and ionization. We also developed the technology for rapid sequencing of short DNA probes which are needed for hybridization diagnosis of mutations. This program is to address the DNA mutation due to the exposure to contaminated media and to promote a better understanding of the relationship between exposure and health impact which are among the top priorities in EMSP program. Mutations of p53 gene is frequently found in fish tumors and certain human cancers. Analysis of these anti-tumor genes in human cancers as well as laboratory induced animal tumors has indicated that particular carcinogens may be responsible for specific types of tumors. However, the direct linkage of each individual contaminant to specific mutation is usually not known. For genes which are highly conserve, the mutation in animal due to the environmental contaminants can have similar effects on human being. By mutation screening of fish in contaminated water, the relationship of mutation and contaminants can be established. The similar relationship can hold true for human being. In this program, we confirmed the conservation of p53 gene between human and medaka fish. We also further found a fish with tumor to have p53 mutation by allele specific polymerase chain reaction mass spectrometry.

The major achievements during the past years are listed and discussed in the follows.

(1) Demonstration of mass resolution improvement with laser induced acoustic desorption:

We developed the innovative approach to use laser induced acoustic wave for DNA desorption. In the past, the use of mass spectrometry for DNA detection is by matrixassisted laser desorption/ionization (MALDI). With MALDI, DNA samples are mixed with small organic compounds. A laser beam with the photon strongly absorbed by the matrix compound but not DNAs is used for desorption and ionization. After the absorption of the laser beam by matrix compounds, the matrix compound gets vaporized and carries DNA molecules into space. The desorbed DNA compounds are subsequently ionized by the protonation or deprotonation process. Although MALDI has had good success in detecting DNAs, the mass resolution is usually poor for large DNA ions due to the broad energy spread caused by the matrix molecules. During the past year, we claerly demonstarted the use of laser induced acoustic desorption to obtain better mass resolution for both protein and DNAs. This technology has great potential for more convenient and reliable DNA mutation analysis for environmental applications. 
We recently developed a new biomolecule detection technology based on an innovative surfaced-assisted desoorption/ionization. The basic principle is some organic compounds on rough surface can get dissociated and give the opportunity to have protonation or deprotonation of biomolecules for desorption and ionization. This invention has been tested successfully for both peptide and oligonucleotides. Since no matrices are needed, we expect the mass resolution can be better. It is also more convenient for hybridization detection. We expect this technology can become a very valuable technology in the future.

(3)

\section{Observation of conservation of p53 gene among human and medaka fish:}

We used allele specific primer for PCR amplification of p53 for both human and medaka fish. We found that high conservation exists among human and medaka fish. It confirms that the measurements of mutation of fish due to the specific contaminant can be used to assess the impact to human being by the same contaminant.We tested exon 5, 6, 7 and 8 in p53 gene and found they are conserved in this region.

(4) Development of matrix-assisted laser desorption/ionization/fragmentation for DNA sequencing.

We developed matrix-assisted laser desorption/ionization with selected fragmentation (MALDIF) for sequencing short DNAs without the need of DNA ladders. We have succeeded in sequencing different primers and probe with the length up to 35 nucleotides. We found that the concentration of ammonia citrate in matrix is a critical factor for selective cleavage for sequencing.

\section{Observation of p53 mutation in medaka fish:}

We have analyzed medaka fish samples from Gulf Coast Laboratory and found mutation in exon 5 in p53 gene for fish with tumors. These fish have been exposed to contaminants. This gives a clear demonstration that the linkage of mutation and contaminants can be established.

\section{Planned activities:}

For the rest of the program, we will try to have automatic sample handling system for multiple samples. We will continue to study the relationship of mutation and contaminant exposure.

\section{Publications during the past year:}

(1) N. I. Taranenko, N. T. Potter, S. L. Allman, V. V. Golovlev and C. H. Chen, "Detection of trinucleotide expansion by Matrix-assisted laser desorption/ionization Time-of-dlight mass spectrometry" Genetic Analysis:Biom\& Eng. 15, 25-31 (1999)

(2) C. H. Chen, V. V. Golovlev, N. I. Taranenko, S. L. Allman, N. R. Isola, N. T. Potter, K. J. Matteson and L. Y. Chang, " Laser desorption mass spectrometry for high-throughput 
DNA analysis and its applications”, Proc. SPIE-Int. Opt. Eng. 3602, 338-345 (1999)

(3) N. R. Isola, S. L. Allman, V. V. Golovlev and C. H. Chen, "Chemical Cleavage Sequencing of DNA using Matrix-assisted Laser Desorption/Ionization Time-of-Flight Mass Spectrometry", Analytical Chemistry 71, 2266-2269 (1999)

(4) V. V. Golovlev, S. S. Lee, S. L. Allman, N. I. Taranenko and C. H. Chen, "Nonresonance MALDI of Oligonucleotides:mechanism of Ion Desorption," Rapid Comm. Mass Spectrom. Analytical Chemistry (in preparation) 